\title{
Enterobactérias isoladas de baratas (Periplaneta americana) capturadas em um hospital brasileiro
}

\author{
Marinésia A. Prado, ${ }^{1}$ Fabiana C. Pimenta, ${ }^{2}$ Miyeko Hayashid, ${ }^{3}$ \\ Paula R. Souza, ${ }^{4}$ Milca S. Pereira ${ }^{1}$ e Elucir Gir ${ }^{5}$
}

RESUMO Objetivo. Isolar e identificar microrganismos em baratas capturadas em um hospital público e determinar o seu perfil de suscetibilidade antimicrobiana.

Métodos. As baratas foram capturadas nos perídos matutino e noturno, colocadas em frascos desinfetados com álcool a 70\%, transferidas para um frasco estéril e levadas ao laboratório. Consideraram-se as baratas integras e vivas, as quais foram colocadas em solução salina estéril $(0,8 \%)$ e homogeneizadas. Essa solução foi semeada nos meios de cultura ágar MacConkey, caldo nutriente, infusão de cérebro e coração (ágar BHI), ágar Sabouraud e ágar manitol. As culturas foram examinadas em um estereomicroscópio para a contagem das unidades formadoras de colônias. Para a determinação do perfil de suscetibilidade antimicrobiana utilizou-se o teste de difusão de disco.

Resultados. Detectou-se prevalência de $56 \%$ de enterobactérias e de $18 \%$ de estafilococos coagulase negativos. Identificaram-se 15 espécies de enterobactérias. As mais freqüentes foram Klebsiella pneumoniae $(17 \%)$; Enterobacter aerogenes $(14 \%)$; Serratia marcescens (13\%); Hafnia alvei (12\%); Enterobacter gergoviae $e$ Enterobacter cloacae (9\%); e Serratia spp. $(6 \%)$. Tanto as enterobactérias quanto os estafilococos coagulase negativos apresentaram uma resistência significativa aos antimicrobianos, inclusive à oxacilina.

Conclusões. A prevalência de bactérias enteropatogênicas e de estafilococos coagulase negativos isolados de baratas Periplaneta americana no hospital estudado demonstra a fragilidade das condutas adotadas tanto para o controle de vetores quanto para o uso dos antimicrobianos. Os resultados demonstram a necessidade da implementação de um programa efetivo de saneamento ambiental e do uso racional dos antimicrobianos dentro das instituições de saúde.

Palavras-chave Infecção hospitalar, contaminação, controle de infecções, insetos vetores.

As baratas, cujo nome vem do latim blatta, que significa inseto que evita a luz, são importantes do ponto de vista sanitário, pois se adaptam a domicílios, hospitais e restaurantes, veicu- lando e disseminando microrganismos $(1,2)$. A Periplaneta americana, também conhecida como barata vermelha, é encontrada no Brasil principalmente nos estados de São Paulo, Amapá, Amazo- nas, Rio Grande do Norte, Bahia e Rio de Janeiro. Atraído pelo cheiro, esse inseto pode chegar ao rosto de pessoas adormecidas para comer detritos alimentares que ficaram impregnados na

\footnotetext{
1 Universidade Federal de Goiás, Faculdade de Enfermagem, Goiânia, Brasil. Correspondência e pedidos de separatas devem ser enviados a Marinésia A. Prado no seguinte endereço: Rua Guatambu 44, Jardim Recreio, CEP 14040-160, Ribeirão Preto, SP, Brasil. E-mail: marinesiadoprado@aol.com
}

2 Universidade Federal de Goiás, Instituto de Patologia e Saúde Pública, Departamento de Microbiologia.

3 Universidade de São Paulo, Escola de Enfermagem de Ribeirão Preto, Ribeirão Preto, Brasil.
4 Universidade de São Paulo, Escola de Enfermagem de Ribeirão Preto, programa de mestrado do Departamento de Enfermagem Geral e Especializada.

5 Universidade de São Paulo, Escola de Enfermagem de Ribeirão Preto, Departamento de Enfermagem Geral e Especializada. 
mucosa bucal. É comum a barata roer os lábios e a região angular da boca de pessoas acamadas inconscientes ou adormecidas, principalmente crianças, quando do regurgitamento do leite, ocasionando no local uma lesão conhecida como herpes blattae (2).

Estudos desenvolvidos nas décadas de 1970 e 1980 identificaram alguns microrganismos associados à infecção hospitalar em baratas capturadas em setores como serviços de nutrição, expurgo do centro cirúrgico e isolamentos. Constatou-se que tais insetos carregam microrganismos em sua superfície durante vários dias, sem que os mesmos percam a sua viabilidade. Dentre os microrganismos isolados de baratas no ambiente hospitalar destacam-se Enterobacter cloacae, Klebsiella pneumoniae, Klebsiella spp., Escherichia coli, Enterobacter aerogenes, Proteus mirabilis, Serratia marcescens, Citrobacter spp., Pseudomonas aeruginosa e Pseudomonas fluorescens (3). A presença de Shigella boydii, Shigella dysenteriae, Salmonella typhmurium, Klebsiella oxytoca e Klebsiella ozaenae em baratas foi verificada por estudos que constataram também que essas estirpes podem desencadear surtos de gastrenterite em pacientes pediátricos (4).

Epidemiologicamente, a barata é um importante vetor no ambiente hospitalar, agravando o flagelo das infecções intra-hospitalares e ambientais. Assim, o objetivo do presente estudo foi isolar e identificar enterobactérias da superfície de baratas capturadas em um hospital público do Centro-Oeste do Brasil e determinar o perfil de suscetibilidade antimicrobiana dos microrganismos isolados.

\section{MATERIAIS E MÉTODOS}

A pesquisa foi realizada de outubro de 1999 a setembro de 2001. O projeto foi aprovado pela Comissão de Ética em Pesquisa da Santa Casa de Misericórdia de Goiânia.

O hospital estudado possui 365 leitos distribuídos nas unidades de internação e conta com ambulatório geral e hemato-oncológico, pronto socorro e serviços de apoio diagnóstico e te- rapêutico. Tem uma média mensal de 15000 atendimentos ambulatoriais e 1278 internações cirúrgicas. O corpo clínico é composto por 186 médicos contratados, 39 médicos residentes e 28 médicos em regime de internato. $\mathrm{O}$ serviço de enfermagem conta com 19 enfermeiros, 105 técnicos e 208 auxiliares de enfermagem. O serviço de nutrição possui dois nutricionistas e 35 auxiliares, enquanto que o serviço de higiene e limpeza é composto por 70 auxiliares de serviços gerais.

\section{Coleta e caracterização das baratas}

As baratas foram capturadas pela manhã e à noite durante 3 semanas consecutivas, 2 dias por semana, nas copas do centro cirúrgico e das enfermarias do segundo andar, onde são internados pacientes do Sistema Único de Saúde (SUS) e pacientes com plano de saúde; na lanchonete que presta serviço terceirizado para o hospital, localizada no primeiro piso; e no serviço de nutrição e dietética. Esses locais foram selecionados porque encontravam-se infestados por baratas no período da coleta.

As baratas foram coletadas por uma das pesquisadoras, que utilizou um frasco previamente descontaminado com água e sabão, seco e desinfetado com álcool a 70\%, friccionado com gaze esterilizada, o qual teve sua base cortada e tampa adaptada. Em seguida, foram transferidas para um frasco esterilizado e transportadas vivas para o laboratório. Somente foram utilizadas para o estudo as baratas que estivessem vivas e íntegras.

As baratas foram imobilizadas a uma temperatura de $0{ }^{\circ} \mathrm{C}$, por um período de 5 a 20 minutos, sendo cada uma delas retirada do frasco com o auxílio de uma pinça anatômica Kelly. A seguir, foram medidas com régua milimétrica e transferidas para um tubo de ensaio contendo $0,5 \mathrm{~mL}$ de solução salina estéril a $0,8 \%$, cujo conteúdo foi homogeneizado rigorosamente por 2 minutos, conforme preconizado por Fotedar (5).

A suspensão salina foi transferida para outro tubo de ensaio, onde foram realizadas diluições decimais de até
$10^{5}$. Alíquotas de $0,25 \mu \mathrm{L}$ foram gotejadas na superfície de placas de Petri contendo ágar MacConkey, de acordo com a técnica descrita por Koneman (6).

As placas de Petri foram incubadas em estufa a $37^{\circ} \mathrm{C}$ de 24 a 48 horas. As colônias bacterianas desenvolvidas foram contadas com o auxílio de um estereomicroscópio, sendo também caracterizadas conforme morfologia macroscópica, submetidas à coloração de Gram e repicadas em ágar inclinado para armazenamento e posterior identificação bioquímica (7).

As colônias isoladas no ágar MacConkey e BHI, caracterizadas como bacilos Gram-negativos, foram submetidas aos seguintes testes: produção de indol, motilidade, produção de sulfeto de hidrogênio, vermelho de metila, utilização do citrato, produção de urease, descarboxilação da fenilalanina desaminase e fermentação de açúcares (glicose, lactose, sacarose e manitol). As reações bioquímicas foram realizadas em tubos de ensaio incubados em estufa a $37^{\circ} \mathrm{C}$ de 24 a 48 horas (7).

\section{Perfil de suscetibilidade antimicrobiana}

Os bacilos Gram-negativos e os cocos Gram-positivos identificados foram submetidos ao teste de difusão de disco seguindo as recomendações do National Committee for Clinical Laboratory Standards (8) para a determinação da suscetibilidade aos antimicrobianos.

As cepas dos bacilos Gram-negativos e Gram-positivos foram semeadas em ágar simples inclinado, incubadas a $37^{\circ} \mathrm{C}$ por 24 horas, sendo o inóculo bacteriano preparado em solução salina a $0,8 \%$, com uma concentração igual à metade da escala 1 de MacFarland (9), e distribuído com o auxílio de uma mecha (swab) na superfície de ágar Muller-Hinton. Os discos contendo os antimicrobianos foram distribuídos de forma eqüidistante, com o auxílio de uma pinça esterilizada.

Os bacilos Gram-negativos foram submetidos aos seguintes antimicrobianos: ampicilina $10 \mu \mathrm{g}$, cefalotina $30 \mu \mathrm{g}$, ceftriaxona $30 \mu \mathrm{g}$, cloranfenicol $30 \mu \mathrm{g}$, gentamicina $10 \mu \mathrm{g}$, sulfazotrim 
TABELA 1. Espécies de enterobactérias isoladas e identificadas de Periplaneta americana em um hospital público, Goiânia (GO), Brasil, 2001

\begin{tabular}{lcr}
\hline \multicolumn{1}{c}{ Microrganismo } & Freqüência & $\%$ \\
\hline K. pneumoniae & 16 & 17 \\
E. aerogenes & 13 & 14 \\
S. marcescens & 12 & 13 \\
Hafnia alvei & 11 & 12 \\
E. cloacae & 8 & 9 \\
E. gergoviae & 8 & 9 \\
Serratia spp. & 6 & 6 \\
Bacilos Gram negativos & 4 & 4 \\
K. oxytoca & 4 & 4 \\
P. vulgaris & 3 & 3 \\
K. ozaeneae & 3 & 3 \\
E. coli & 2 & 2 \\
K. rhinoscleromatis & 2 & 2 \\
Enterobacter & 1 & 1 \\
M. morganii & 1 & 1 \\
\multicolumn{1}{c}{ Total } & 94 & \\
\hline
\end{tabular}

TABELA 2. Freqüência e percentual de unidades formadoras de colônia das enterobactérias isoladas de Periplaneta americana de um hospital público, Goiânia (GO), Brasil, 2001

\begin{tabular}{rrrcr}
\hline Unidades formadoras de colônias $^{\mathrm{a}}$ & Freqüência & $\%$ \\
\hline 10 & a & 10000 & 54 & 59 \\
10000 & $\mathrm{a}$ & 20000 & 9 & 10 \\
20000 & $\mathrm{a}$ & 30000 & 7 & 8 \\
30000 & $\mathrm{a}$ & 40000 & 5 & 5 \\
40000 & $\mathrm{a}$ & 50000 & 7 & 8 \\
50000 & $\mathrm{a}$ & 60000 & - & - \\
60000 & $\mathrm{a}$ & 70000 & 1 & 1 \\
70000 & $\mathrm{a}$ & 80000 & - & - \\
80000 & $\mathrm{a}$ & 90000 & - & - \\
90000 & a & 100000 & 6 & 6 \\
100000 & a & 200000 & 3 & 3 \\
Total & & & 92 & 100 \\
\hline
\end{tabular}

${ }^{a}$ Houve crescimento de colônias distintas em uma mesma barata.

$250 \mu \mathrm{g}$, cefepime $30 \mu \mathrm{g}$, vancomicina $30 \mu \mathrm{g}$, aztreonam $30 \mu \mathrm{g}$, ampicilina/ sulbactam $30 \mu \mathrm{g}$, amicacina $30 \mu \mathrm{g}$ e ciprofloxacina $5 \mu \mathrm{g}$. Os bacilos Grampositivos foram submetidos a cefalexina $30 \mu \mathrm{g}$, vancomicina $30 \mu \mathrm{g}$, ampicilina $10 \mu \mathrm{g}$ e oxacilina $1 \mu \mathrm{g}$.

Os resultados foram analisados em parceria com a equipe de pesquisadores do Departamento de Microbiologia, Imunologia, Parasitologia e Patologia do Instituto de Patologia Tropical e Saúde Pública, Universi- dade Federal de Goiás. Foram calculados freqüência, percentual, moda e mediana. Para a análise estatística utilizou-se o programa Epi-Info versão $6.04(10)$.

\section{RESULTADOS}

Das 103 baratas analisadas, coletadas em cinco unidades distintas do hospital, 91 apresentaram crescimento bacteriano. A freqüência de microrga- nismos foi: $97 \%$ de fungos; $56 \%$ de enterobactérias; $18 \%$ de estafilococos coagulase negativos e 3\% de bacilos Gram negativos.

Foram isoladas 94 cepas de enterobactérias, categorizadas em 15 espécies (tabela 1). O número de unidades formadoras de colônias (ufc) encontradas por barata variou de 10 a 200000 (tabela 2). A mediana e a moda verificadas para o grupo das enterobactérias foram ambas de $100000 \mathrm{ufc/barata.} \mathrm{A}$ tabela 3 mostra a suscetibilidade antimicrobiana das enterobactérias isoladas; a tabela 4 mostra a suscetibilidade antimicrobiana dos estafilococos coagulase negativos. A tabela 5 mostra a suscetibilidade antimicrobiana segundo a espécie de enterobactéria. Os fungos não foram submetidos ao teste de suscetibilidade antimicrobiana.

\section{DISCUSSÃO}

A Klebsiella pneumoniae foi a espécie mais prevalente nas baratas coletadas, seguida por Enterobacter aerogenes, Serratia marcescens, Hafnia alvei, Enterobacter cloacae e Enterobacter gergoviae. Todas essas enterobactérias podem ser associadas a surtos e epidemias hospitalares. A determinação do perfil de suscetibilidade das enterobactérias aos antimicrobianos permitiu a observação de cepas resistentes à maioria dos antimicrobianos, e de estafilococos coagulase negativos resistentes à oxacilina, cefalexina e ampicilina. No Brasil, a resistência à amicacina tem sido observada em 20 e $30 \%$ das amostras de Escherichia coli, Enterobacter, Klebsiella spp. e Proteus (11). K. pneumoniae e E. coli são freqüentemente isolados em bacteriemias e infecções associadas a cateteres vasculares, com elevado perfil de resistência (12). Os dados apresentados alertam para a necessidade de padronização e uso criterioso dos antimicrobianos dentro das instituições de saúde, bem como da prescrição e do uso restrito desses medicamentos na comunidade em geral.

Em estudo recente, também no Brasil, acerca do perfil de resistência de microrganismos isolados de 635 hemoculturas de neonatos, verificou-se um 
TABELA 3. Suscetibilidade antimicrobiana das enterobactérias isoladas de Periplaneta americana em um hospital público, Goiânia (GO), Brasil, 2001

\begin{tabular}{|c|c|c|c|c|c|c|c|c|}
\hline \multirow[b]{2}{*}{ Suscetibilidade } & \multicolumn{2}{|c|}{ Sensível } & \multicolumn{2}{|c|}{ Intermediário } & \multicolumn{2}{|c|}{ Resistente } & \multicolumn{2}{|c|}{$\mathrm{T}^{\mathrm{a}}$} \\
\hline & $\mathrm{F}^{\mathrm{b}}$ & $\%$ & $\mathrm{~F}$ & $\%$ & $\mathrm{~F}$ & $\%$ & $\mathrm{~F}$ & $\%$ \\
\hline Ampicilina & 15 & 16 & 6 & 7 & 72 & 77 & 93 & 100 \\
\hline Amicacina & - & - & 2 & 33 & 4 & 67 & 6 & 100 \\
\hline Aztreonam & - & - & 3 & 50 & 3 & 50 & 6 & 100 \\
\hline Cefalotina & 23 & 25 & 5 & 5 & 65 & 70 & 93 & 100 \\
\hline Ciprofloxacina & 6 & 100 & - & - & - & - & 6 & 100 \\
\hline Cloranfenicol & 65 & 70 & 2 & 2 & 26 & 28 & 93 & 100 \\
\hline Gentamicina & 3 & 3 & 15 & 16 & 75 & 81 & 93 & 100 \\
\hline Sulfazotrim & 77 & 83 & 1 & 1 & 15 & 16 & 93 & 100 \\
\hline
\end{tabular}

a $T$ = número de cepas de enterobactérias submetidas ao antibiograma.

${ }^{b} \mathrm{~F}=$ freqüência.

TABELA 4. Suscetibilidade dos estafilococos coagulase negativos isolados de Periplaneta americana capturadas em um hospital público, Goiânia (GO), Brasil, 2001

\begin{tabular}{|c|c|c|c|c|c|c|c|c|}
\hline \multirow[b]{2}{*}{ Antimicrobiano } & \multicolumn{2}{|c|}{ Sensível } & \multicolumn{2}{|c|}{ Intermediário } & \multicolumn{2}{|c|}{ Resistente } & \multicolumn{2}{|c|}{$\mathrm{T}^{\mathrm{a}}$} \\
\hline & $\mathrm{F}$ & $\%$ & $\mathrm{~F}$ & $\%$ & $\mathrm{~F}$ & $\%$ & $F$ & $\%$ \\
\hline Ampicilina & 8 & 62 & 1 & 1 & 4 & 31 & 13 & 100 \\
\hline Cefepime & 10 & 77 & - & - & 3 & 23 & 13 & 100 \\
\hline Oxacilina & 5 & 39 & 3 & 23 & 5 & 39 & 13 & 100 \\
\hline Vancomicina & 13 & 100 & - & - & - & - & 13 & 100 \\
\hline
\end{tabular}

a $T$ = número de cepas de estafilococos coagulase negativos submetidas ao antibiograma.

TABELA 5. Suscetibilidade antimicrobiana das enterobactérias isoladas de Periplaneta americana segundo a espécie em um hospital público de Goiânia (GO), Brasil, 2001 a

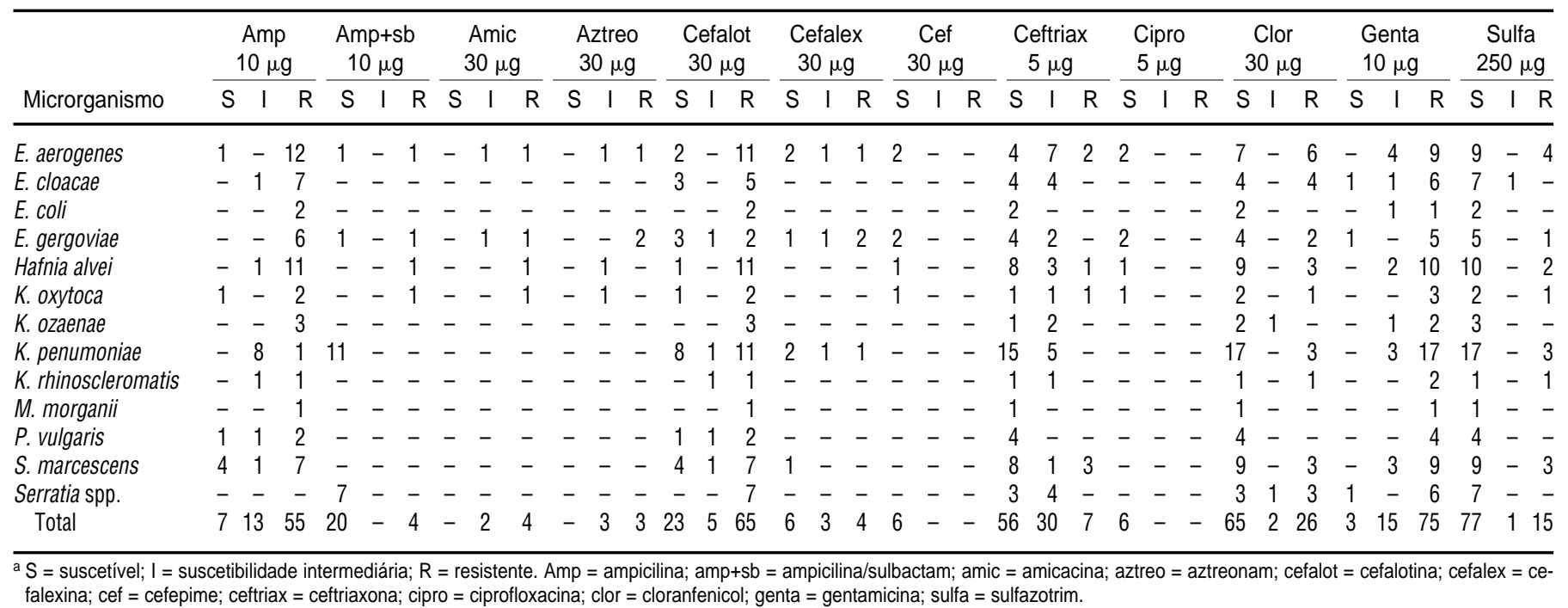


percentual de $65 \%$ de bacilos Gramnegativos (13). Dentre eles, destacaram-se $36 \%$ de K. pneumoniae e $13 \%$ de S. marcescens. Ainda no mesmo estudo, os Gram-positivos mais freqüentes foram os estafilococos coagulase negativos $(65 \%)$. Os Gram-negativos apresentaram uma média de resistência de $82 \%$ para o cloranfenicol; $74 \%$ para sulfametoxazol-trimetoprim; $66 \%$ para as cefalosporinas; $65 \%$ para os aminoglicosídeos; $53 \%$ para tetraciclinas; $7 \%$ para ciprofloxacina e 3\% para imipenem. Quanto aos Gram-positivos, destacaram-se $64 \%$ de Staphylococcus aureus resistentes à meticilina (13).
A presença de baratas e de microrganismos como os encontrados na pesquisa não é um problema que aflige somente os hospitais brasileiros. No tocante às medidas de controle com vistas a diminuir o número de baratas, já que as mesmas não podem ser eliminadas, é importante destacar que condições climáticas como umidade e temperatura elevada favorecem a proliferação das baratas. Além disso, os alimentos devem ser mantidos em recipientes fechados; o ambiente deve ser mantido livre de fragmentos e restos de alimentos ou matéria orgânica; as lixeiras devem ficar fechadas e devem ser esvaziadas com freqüência; a estrutura física deve ser continuamente monitorada, e as frestas em paredes, portas e rodapés, assim como as rachaduras nos sistemas de distribuição de água, esgoto e eletricidade, devem ser vedadas (1-4).

A problemática das baratas pode ser minimizada, desde que haja um processo de conscientização entre os profissionais de saúde e os administradores das instituições hospitalares no que tange à implementação de um programa efetivo de saneamento ambiental que seja adotado por todos de forma sistematizada.

\section{REFERÊNCIAS}

1. Luckmann WH, Metcalf RL. The pestmanagement concept. Em: Metcalf RL, Luckmann WH, eds. Introduction to insect pest management. New York: John Wiley; 1982. Pp. 1-31.

2. Rozendaal FG. Vector control methods for use by individuals and communities. Geneva: World Health Organization; 1997. Pp. 288-301.

3. Rivault C, Cloarec A, Leguyader A. Bacterial load of cockroaches in relation to urban environment. Epidemiol Infect 1993;110(2):317-325.

4. Oothuman P. Bacterial pathogens isolated from cockroaches trapped from paediatric wards in peninsular Malaysia. Trans $\mathrm{R}$ Soc Trop Med Hygiene 1989;83(1):133-135.

5. Fotedar R, Shriniwas UB, Verna A. Cockroaches (Blattella germanica) as carriers of microorganisms of medical importance in hospitals. Epidemiol Infect 1991;107(1): 181-187.

6. Westergren G, Krasse B. Evaluation of a micromethod for determination of Strep- tococcus mutans and Lactobacillus infection. J Clin Microbiol 1978;7(1):82-83.

7. Koneman EW, Allen SD, Dowell VRJ Sommers HM. Diagnóstico microbiológico - texto e atlas colorido. 2a ed. São Paulo: Panamericana; 1993.

8. National Committee for Clinical Laboratory Standards. Performance standards for antimicrobial disk susceptibility tests; approved standard-seventh edition. M2A7. Wayne, PA: NCCLS; 2000.

9. Bier O. Microbiologia e imunologia. $24^{a}$ ed. São Paulo: Melhoramentos; 1985. Pp. 930-931.

10. Dean AG, Dean JA, Coulombier D, Brendel KA, Smith DC, Burton AH, et al. Epi Info, Version 6.04: a word-processing database, and statistics program for public health on IBM-compatible microcomputers. Atlanta, Georgia: Centers for Disease Control and Prevention; 1997.

11. Gomes ACLF, Martinez R. Detecção de enterobactérias produtoras de betalactamases de espectro estendido (ESBL) em amostras de sangue, cateter e outros materiais biológicos de pacientes do hospital das Clínicas de Ribeirão Preto (HCFMRP). Em: $21^{\circ}$ Congresso Brasileiro de Microbiologia; 2001 out 21-25; Foz do Iguaçu, Paraná. Rio de Janeiro: Armazém da Letra; 2001.

12. Moraes BA. Perfil de resistência bacteriana em hemoculturas positivas de recém-natos em uma maternidade do Rio de Janeiro no período de 1997 a 2001. Em: 21 Congresso Brasileiro de Microbiologia; 2001 out 21-25; Foz do Iguaçu, Paraná. Rio de Janeiro: Armazém da Letra; 2001. Pp. 158.

13. Tavares W. Manual de antibióticos e quimioterápicos antiinfecciosos. São Paulo: Atheneu; 1999.

Manuscrito recebido em 27 de novembro de 2001. Aceito em 26 de dezembro de 2001. 
ABSTRACT Objective. To isolate and identify microorganisms from cockroaches that were captured in a public hospital and to test the antimicrobial susceptibility of these microorganisms.

\title{
Enterobacteria isolated from cockroaches (Periplaneta americana) captured in a Brazilian hospital
}

Methods. Cockroaches were captured in the morning and at night. They were placed in flasks rinsed with 70\% alcohol, transferred to sterilized flasks, and then taken to the laboratory. Only cockroaches captured whole and live were utilized for the study. After being immobilized at 0 degrees $C$, each cockroach was placed in a test tube with sterile saline solution $(0.8 \%)$ and then homogenized. The resulting solution was then placed in the following five culture media: MacConkey agar, nutrient broth, brain-heart infusion agar, Sabouraud agar, and mannitol. The cultures were examined using a stereomicroscope, and colony-forming units were counted. The disk diffusion test was used to determine antimicrobial susceptibility.

Results. We found a $56 \%$ prevalence of enterobacteria and an $18 \%$ prevalence of coagulase-negative staphylococci. Fifteen species of enterobacteria were identified. The most frequent were Klebsiella pneumoniae (17\%), Enterobacter aerogenes (14\%), Serratia marcescens (13\%), Hafnia alvei (12\%), Enterobacter gergoviae and Enterobacter cloacae (each 9\%), and Serratia spp. (6\%). Both the enterobacteria and the coagulase-negative staphylococci showed significant resistance to antimicrobials, including oxacillin. Conclusions. The prevalence of enteropathogenic bacteria and coagulase-negative staphylococci isolated from Periplaneta americana cockroaches in the studied hospital reflects the weakness of the measures adopted both for vector control and for antimicrobial use. The results show the need to implement effective health-institution programs focusing on hygiene and the rational use of antimicrobials.

\section{Fifth Annual Conference on Vaccine Research}

\author{
Dates: $\quad$ 6-8 May 2002 \\ Location: Wyndham Baltimore Inner Harbor Hotel \\ Baltimore, Maryland, United States of America
}

The Fifth Annual Conference on Vaccine Research will focus on three areas: basic science, product development, and clinical and field studies. The meeting is sponsored by the National Foundation for Infectious Diseases, in collaboration with the World Health Organization, the Centers for Disease Control and Prevention of the United States, and other groups.

A multidisciplinary forum, the conference will feature such activities as leading international experts participating in seminars and panel discussions on basic immunology, vaccine development, clinical testing, and vaccine regulations.

The meeting is intended for persons with an interest in and knowledge of vaccine research, including researchers and scientists, molecular biologists, epidemiologists, public health officials, microbiologists, physicians, and vaccine manufacturers. The fee for the conference (not including hotel accommodations and meals) is US\$ 350 if paid by March 22, and US\$ 400 after that date.

\author{
Information: \\ National Foundation for Infectious Diseases \\ 4733 Bethesda Avenue, Suite 750 \\ Bethesda, Maryland 20814-5278, United States of America \\ Telephone: (301) 656-0003, extension 19 \\ Fax: (301) 907-0878 \\ E-mail: vaccine@nfid.org. \\ Internet: http://www.nfid.org/conferences/vaccine02/
}

